Roderick B. De Castro, MD

Michelle Angelica B. Cruz-Daylo, MD

Monique Lucia A. Jardin, MD

Department of Otorhinolaryngology

Head and Neck Surgery

Ospital ng Makati

\section{Low Frequency Ultrasound in Chronic Rhinosinusitis with Nasal Polyposis and Recovery after Endoscopic Sinus Surgery: A Randomized Controlled Trial}

\begin{abstract}
Objective: The study aimed to determine the role of low frequency ultrasound in patients with Chronic Rhinosinusitis with Nasal Polyposis (CRS-NP) and recovery after Endoscopic Sinus Surgery (ESS) using Sino Nasal Outcome Test 22 (SNOT-22) questionnaires, modified Lund MacKay endoscopic appearance and histopathologic examination.
\end{abstract}

\section{Methods:}

$\begin{array}{ll}\text { Design: } & \text { Single Blinded Randomized Controlled Trial } \\ \text { Setting: } & \text { Tertiary Government Hospital }\end{array}$

Participants: 42 adult Filipinos aged 19 to 76 years old diagnosed with Chronic Rhinosinusitis with grade 2 and 3 Nasal Polyposis and failure of maximal medical management (3-month course of antibiotics, nasal douche, topical steroids and other modalities) between June 2013 to June 2015 were randomized into two groups of 21 participants each-- the ultrasoundtreated group and control group. Specimens (nasal polyps) from both groups were obtained and processed with Hematoxylin-Eosin (H\&E) and gram staining. Specimens from the ultrasoundtreated group received low frequency ultrasound $\left(1 \mathrm{MHz}, 1.0\right.$ watt $/ \mathrm{cm}^{2}, 20 \%$ pulsed mode for 5 minutes at $37^{\circ} \mathrm{C}$ ) post-extraction and prior to staining. In phase II, the ultrasound group also received the same ultrasound treatment while the control group underwent ultrasound at $0 \mathrm{MHz}$ frequency, 0 watt $/ \mathrm{cm}^{2}$, both twice a week for 3 weeks, beginning one (1) week post operatively. Both groups accomplished SNOT-22 forms and were evaluated via modified Lund MacKay endoscopic appearance at 1 week (week 0 of treatment), 2 weeks, 3 weeks, and 1 month post operatively (week 3 of treatment).

Results: Paired T-test showed a statistically significant difference between control and treatment groups in epithelial thickness with a p-value of 2.29E-10 (average of 73.34um for controls and 31.1 um for the treatment group) at 95\% confidence interval. The inflammatory cell count also differed significantly between control and treatment groups (average 293.85 and 29.65 inflammatory cells per high-power field in 10 random microscopic fields, respectively), p-value of $1.05 \mathrm{E}-17$ on paired T-test; Cl 95\%. In phase II of the study, SNOT-22 results showed significant differences in improvement of symptoms in ultrasound-treated patients after Endoscopic Sinus Surgery (weekly mean scores of 38.05, 21, 11.3, and 10.45) and in modified Lund Mackay endoscopic appearance scores (weekly mean scores of 7.88, 4.35, 3.02, 2.08). Two-way analysis of variance showed significant differences between control and treatment groups for both 
SNOT-22 ( $p=1.07 \mathrm{E}-80 ; 9.71 \mathrm{E}-119 ; \mathrm{Cl} 95 \%$ ) and modified Lund Mackay endoscopic appearance scores $(p=3.89 \mathrm{E}-60 ; 1.85 \mathrm{E}-95 ; \mathrm{Cl} 95 \%)$.

Conclusion: Low frequency therapeutic ultrasound demonstrated possible efficacy as an agent in disrupting epithelial architecture in patients with CRS-NP as well as in symptom improvement after endoscopic sinus surgery patients based on histopathologic evaluation, SNOT-22 and modified Lund MacKay endoscopic appearance scores. Low frequency ultrasound may be an adjuvant to conventional medical treatment in CRS-NP.

Keywords: Biofilm, Sinusitis, Nasal Polyps, Chronic disease, Ultrasonic therapy

Chronic Rhinosinusitis (CRS) is an inflammation of the nasal cavity and paranasal sinuses that has been present for at least 12 weeks characterized by: (1) nasal congestion, obstruction or blockage with (2) facial pain or pressure, (3) discolored discharge, or (4) hyposmia or anosmia. ${ }^{1}$ Nasal polyposis (NP) is a subgroup of CRS.' Chronic Rhinosinusitis with Nasal Polyposis (CRS-NP) is considered an immunological disease affected by immunological disorders, environmental factors, Staphylococcus superantigens, fungal infections and the presence of microbial biofilms which lead to its chronicity and/or recurrences especially in patients with biofilm formation. ${ }^{2}$ Biofilms are highly organized communities of bacteria which produce a protective extracellular matrix against host defense and antibiotics. ${ }^{3}$ Hypotheses explaining reduced susceptibility of biofilms are poor antimicrobial penetration, deployment of adaptive stress responses, physiological heterogeneity in the biofilm population, and the presence of phenotypic variants or persister cells. ${ }^{3}$ Traditionally, biofilms are detected using electron microscope and confocal scanning electron microscope. ${ }^{4}$ However, some studies showed correlation of histopathological evaluation using gram stain and hematoxylin-eosin staining in detecting biofilm in the sinonasal discharge of patients with CRS. ${ }^{5}$ The presence of a biofilm in H\&Estained slides was diagnosed on the following criteria (1) irregularly shaped groupings of small basophilic material one third of the size of the surrounding epithelial or inflammatory cells; (2) presence of a biofilm over the epithelial lining, not in or under the epithelial lining; (3) biofilm tightly adherent to the surface epithelium or pulled away slightly; or (4) a dense extracellular polysaccharide substance (EPS) material with embedded basophillic substance coating the epithelial surface. $^{5}$

The current treatment for CRS focuses on the use of steroids and antimicrobials. ${ }^{6}$ With the premise of biofilm presence, alternative therapeutic strategies are being explored to block inter-bacterial molecular communication, inhibiting biofilm production and disrupting bacterial biofilms. ${ }^{7}$ Proposed interventions include the use of biocides like manuka honey and vitamin $\mathrm{D}$, chelating agents and biophysical agents such as low frequency therapeutic ultrasound. Ultrasound consists of waves (greater than 20,000 cycles per second) that transmit energy by alternating compressing and rarefying material to the crystal (piezoelectric properties) in the transducer of an ultrasound machine. ${ }^{9}$ Waves produced at therapeutic level (1-3MHZ) by pulsed ultrasound acts on a cellular and molecular level which alters membrane permeability and ionic concentration gradients thus changing cellular biochemical activity. ${ }^{9}$ Hseieh et al. demonstrated that pulsed ultrasound decreased the number of nitric oxide synthase-producing neurons in rats with induced inflammatory arthritis therefore decreasing pain in inflammatory conditions. ${ }^{10}$

In CRS, ultrasound may cause a bioaccoustic effect, breaking down biofilms directly by killing or reducing the viability of bacteria, making them accessible to antibiotic intervention ${ }^{11}$ This non-thermal effect of ultrasound increases blood flow to the area and decreases edematous swelling resulting in microcavitation and acoustic streaming which produces vibration and subsequent transmission of ultrasound energy to the cell membrane, vascular walls and biofilm. ${ }^{9}$ Young et al., reported that use of $1 \mathrm{MHz}$ therapeutic ultrasound caused worsening of symptoms in $10 \%$ of participants with CRS with total mean percentage improvement of overall symptom score of $16.7 \%$ (Wilcoxon test) and $34 \%$ improvement in 20-item Sino-Nasal Outcome Test. ${ }^{11}$

This study aimed to determine the role of low frequency ultrasound in patients with CRS-NP via histopathological analysis (H\&E and Gram staining) and in recovery after ESS using subjective assessment of the Quality of Life using SNOT-22 questionnaire as well as objective assessment of the nasal mucosal recovery and endoscopic appearance via Modified Lund MacKay endoscopic appearance.

\section{METHODS}

With ethics committee approval, this single blinded randomized controlled trial was conducted at a tertiary public training hospital from June 1, 2013 to June 30, 2015. Written informed consent was obtained from all participants.

\section{Subjects/ sampling}

A sample size of 20 was computed based on the institutional outpatient department annual CRS census using the formula $n=t^{2} x$ $\mathrm{p}(1-\mathrm{p}) / \mathrm{m}^{2}$ (base sample size calculation) with a type 1 error of 0.05 and type 2 error of $95 \%$ and a prevalence of $1.35 \%$ of total OPD consults. Considered for inclusion were adult patients aged 19 and above who satisfied the operational definition of Chronic Rhinosinusitis with Grade 2 or 3 Nasal Polyposis and were recommended to undergo functional endoscopic sinus surgery based on Philippine Society of Otolaryngology - Head and Neck Surgery (PSO-HNS) Clinical Practice Guidelines; ; who had undergone maximal medical management (more than 3 month course of antibiotics, nasal douche, topical steroids and 


\section{ORIGINAL ARTICLES}

other modalities) with no appreciable improvement; and had evidence of sinus mucosal disease and nasal polyps demonstrated on sinus CT imaging and nasal endoscopy. Those who had chronic diseases or co-morbidities (diabetes, hypertension), were immunocompromised, pregnant, had sinonasal malignancy, known ciliary disorder, skin diseases of the face (acne), other facial skin lesions or allergies, and contraindications for ultrasound (thrombophlebitis, pacemaker, fracture with titanium implants) were excluded from the study.

\section{Randomization/ Blinding}

Participants were randomly divided into 2 groups, ultrasoundtreated and control, using a simple randomization technique. A total of 21 patients were allocated to each group. Assignment was made by a research assistant (ORL resident) other than the researchers by providing written codes designating control or intervention. These papers were placed in a folded sheet of foil inside the envelope. There was no detectable difference in size or weight between intervention and control envelopes. Envelopes used were colored brown and were opened sequentially only after writing subjects tracking information on the envelope.

\section{Procedure and data analysis}

Histories were obtained and otorhinolaryngological examinations performed by two ORL residents. All participants had routine laboratory parameters (chest $x$-ray, complete blood count, urinalysis, prothrombin and partial thromboplastin time, fasting blood sugar, blood typing) checked pre-operatively. All underwent early pre-operative anesthesia evaluation. Patients more than 34 years old also underwent medical risk assessment.

The study was divided in 2 phases. The first phase determined the role of low frequency ultrasound on nasal polyps via histopathological analysis (Hematoxylin - Eosin and Gram stain). The second phase determined the effect of ultrasound treatment on recovery among participants after ESS using SNOT-22 questionnaire and monitoring mucosal recovery using modified Lund MacKay endoscopic appearance.

\section{Phase 1}

All procedures were performed under inhalational general endotracheal anesthesia using sevoflorane. Six different surgeons by rotation performed ESS using the Messerklinger technique with cutting and grasping forceps without a microdebrider. Different anesthesiologists administered the same anesthesia protocol for each patient.

Nasal polyps that were analyzed from both arms measured at least $2 \mathrm{~cm} \times 2 \mathrm{~cm}$ or larger and were extracted carefully by Blakesly forceps at the root or $5 \mathrm{~mm}$ from the root of the polyp to avoid iatrogenic injury to the mucosal surface of the specimens. After extraction, specimens were immediately placed in a laboratory beaker containing $0.9 \%$ sodium chloride solution. Specimens from the control group were sent directly to the laboratory for tissue processing and staining while specimens from the ultrasound group went directly to the physical therapy department for treatment. All specimens from the treatment group received ultrasound therapy from the same machine (Metron Accusonic Plus Ultrasound Therapy unit Model AP 170, Balkowitsch Enteprises Inc; Chicago IL) using $3.5 \mathrm{~cm}$ diameter probe within 30 minutes of sampling. Pulsed ultrasound (20\%) treatment with 1 $\mathrm{MHz}$ frequency and 1 watt $/ \mathrm{cm}^{2}$ sound pressure was employed for 5 minutes at $37^{\circ} \mathrm{C}$ in $100 \mathrm{ml}$ total volume of $0.9 \%(\mathrm{w} / \mathrm{v})$ sodium chloride solution. A distance of approximately $2 \mathrm{~cm}$ was maintained between the ultrasound probe and the specimen. A digital thermometer was applied to register any temperature changes during treatment. Specimens were also sent to the laboratory after treatment. All specimens were fixed in $10 \%(\mathrm{w} / \mathrm{v})$ formaldehyde one hour after sampling. Specimens were processed for conventional staining with Hematoxylin and Eosin and Gram staining according to institutional standard operating procedures.

The criteria for the histopathological detection of microbial biofilms were the presence of characteristic morphology: (1) irregularly shaped groupings of small basophilic material one third of the size of the surrounding epithelial or inflammatory cells; (2) presence of a biofilm over the epithelial lining, not in or under the epithelial lining; (3) biofilm tightly adherent to the surface epithelium or pulled away slightly; or (4) a dense extracellular polysaccharide substance (EPS) material with embedded basophillic substance coating the epithelial surface. ${ }^{5}$ Due to technical difficulties and in absence of electron microscope for standard comparison, only the epithelial thickness (structural) and number of inflammatory cells per $10 \mathrm{HPF}$ (epithelial and subepithelial cellular infiltration) were studied to correlate the effect of ultrasound on the architectural integrity of nasal polyps between the two groups.

The histopathological characteristics of biofilms and epithelial and subepithelial layers in control group specimens were compared to ultrasound-treated nasal polyps. The number of inflammatory cells per high power field in 10 random microscopic fields were counted and recorded. Histological examinations were performed by blinded pathologists; two residents in training supervised by a board-certified consultant.

\section{Data analysis for phase 1}

Collated results were tabulated. Data were entered on Microsoft Excel spreadsheets (Microsoft Office 2013, Microsoft Corporation, WA, USA) and analyzed per criterion. Statistical analysis utilized Student T test to compare control and treatment groups with 95\% confidence interval and level of significance set at $p<0.05$ and 2-way ANOVA to analyze effects of time and treatment. 


\section{Phase II}

Patients' symptoms were graded initially according to the 22 -item Outcome Test (SNOT-22).

We used the modified Lund-MacKay score of endoscopic appearance to assess the following endoscopic parameters: polypoid appearance, edema, presence of granulation, presence of discharge, adhesion, and crusting. Each parameter was graded 0 (absent), 1 (mild), 2 (moderate) or 3 (severe), according to endoscopic appearance ${ }^{16}$ each side could have a minimum score of 0 and a maximum score of 18 . Nasal endoscopy was performed by two ORL residents with the guidance of a board-certified ORL Consultant using 0 degree and 30 degree Hopkins endoscopes (Karl Storz GmbH \&Co., Germany). Evaluators were blinded regarding group assignment of the participants.

The ultrasound-treated group received the same ultrasound treatment used in phase 1 for a total of 6 treatments, 2 sessions per week, 3 and 4 days apart, for 3 weeks, beginning 1 week post operatively. In sitting position, water-based ultrasound gel (Biocare Medical System Inc, Makati Philippines) was applied over the skin overlying the maxillary sinuses and frontal sinus, and the area of the nasal dorsum in a circular pattern and a 1-MHz pulsed low-intensity ultrasound probe (Metron Accusonic Plus Ultrasound Therapy unit Model AP 170, Balkowitsch Enteprises Inc; Chicago IL) was applied systematically, moving in circular patterns over the areas mentioned above within a period of 5 minutes. The control group also underwent ultrasound treatment with the following parameters $(0 \mathrm{~Hz}, 0 \mathrm{~W} / \mathrm{cm}-2$, $20 \%$ pulsed mode, for 5 minutes). They were all asked to accomplish Sino-Nasal Outcome Test (SNOT-22) questionnaires once a week for 4 weeks during pre treatment (week 0 of treatment/ 1 week post op), and weeks 1,2 and 3 of treatment (weeks 2, 3 and 4 post-op). All participants also started using sodium chloride nasal spray (2 sprays per nostril thrice daily) and topical steroids (Fluticasone propionate $50 \mathrm{mcg}$ nasal spray, UNILAB, Philippines) 2 sprays per nostril once a day 1 week post operatively. All participants were compliant with the medications and follow up sessions.

\section{Data analysis for phase II}

Collated results were tabulated and data was entered on Microsoft Excel (Microsoft Office 2013, Microsoft Corporation, Washington) spreadsheets. Data was described in terms of means and percentage. Statistical Analysis used Student $T$ test to compare control and ultrasound treated groups with level of significance set at $p<0.05$ (confidence interval of 95\%) and a 2-way ANOVA to analyze effects of time and treatment.

\section{RESULTS}

A total of 42 patients consisting of 19 males and 23 females (mean age $=42.8$ years; range 19-72 years) were recruited and consented. Forty-one (41) participants with CRS-NP completed this study. One subject from the treatment group was withdrawn during phase 1 of the study because of a final histopathology result of inverted papilloma (withdrawal rate of 2.38\%). There were no dropouts. Thirty-one (76\%) had grade II Nasal Polyps and 10 (24\%) had grade III nasal polyps. Eight patients (20\%) had previous Endoscopic Sinus Surgery with one of them having had 2 prior surgeries. All patients were treated with topical fluticasone propionate $50 \mathrm{mcg}$ nasal spray 2 sprays both nostrils bid prior to surgery. Mean average use of intranasal topical steroids was 6.2 months with range from 4 to 10 months.

\section{Phase 1}

All histopathology results from this study showed inflammatory polyps with infiltration of eosinophils, neutrophils and granulocytes except for one specimen in the treatment group that showed inverted papilloma. In the control group, 18 specimens (86\%) showed average epithelial thickness (surface epithelium + biofilm thickness) of 73.34um (range 33 um to 115.2 um). (Figure 1) Of these 18 specimens, two (9.5\%) showed areas with stratified squamous epithelium metaplasia while three (14\%) out of total number of specimens in control showed normal architecture. The treatment group specimens showed an average epithelial thickness (surface epithelium + biofilm thickness) of 31.18um with range from $19.3 u m$ to $45.2 u m$. (Figure 2) The paired T-test revealed a statistically significant difference between control and treatment groups ( $p=2.29 \mathrm{E}-10 ; \mathrm{Cl} 95 \%)$. (Figure 3 )

Of an average of 10 high power objective magnification microscopic fields, the control group showed 293.86 inflammatory cells (range 174 to 480), mostly neutrophils, granulocytes and eosinophils. The ultrasound treated group had 29.65 inflammatory cells (7 to 48) per 10 HPO magnification fields. The paired T-test showed a statistically significant difference between control and treatment groups $(p=$ 1.05E-17; Cl 95\%). (Figure 4)

\section{Phase 2}

SNOT-22 mean score control group results for weeks 0 (1week post op), 1, 2, and 3 were 38.29 (SD 1.62); 37.48 (SD 1.36); 28.1 (SD 1.73) and 16.57 (SD 1.78) respectively. Paired T-test scores showed no statistically significant difference between week 0 and week 1 scores in the control group. However, there was a statistically significant difference in symptom regression for weeks 2 and 3 in the control group. (Table 1)

SNOT-22 mean scores for the ultrasound treated group were 38.05 (SD 1.96), 21 (SD 1.49), 11.3 (SD 1.59), and 10.45 (1.19) for week 0 to week 3 , respectively. There was greatest decrease in mean scores between week 0 and week 1 (from 38.05 to 21). The paired T-test revealed a statistically significant difference in means of the treated group per week especially at week 1 and week 2 of treatment. (Table 1)

Comparing SNOT-22 results between two groups showed the greatest difference occurred after week 1 and week 2 of treatment. The Student T-test revealed a statistically significant difference 

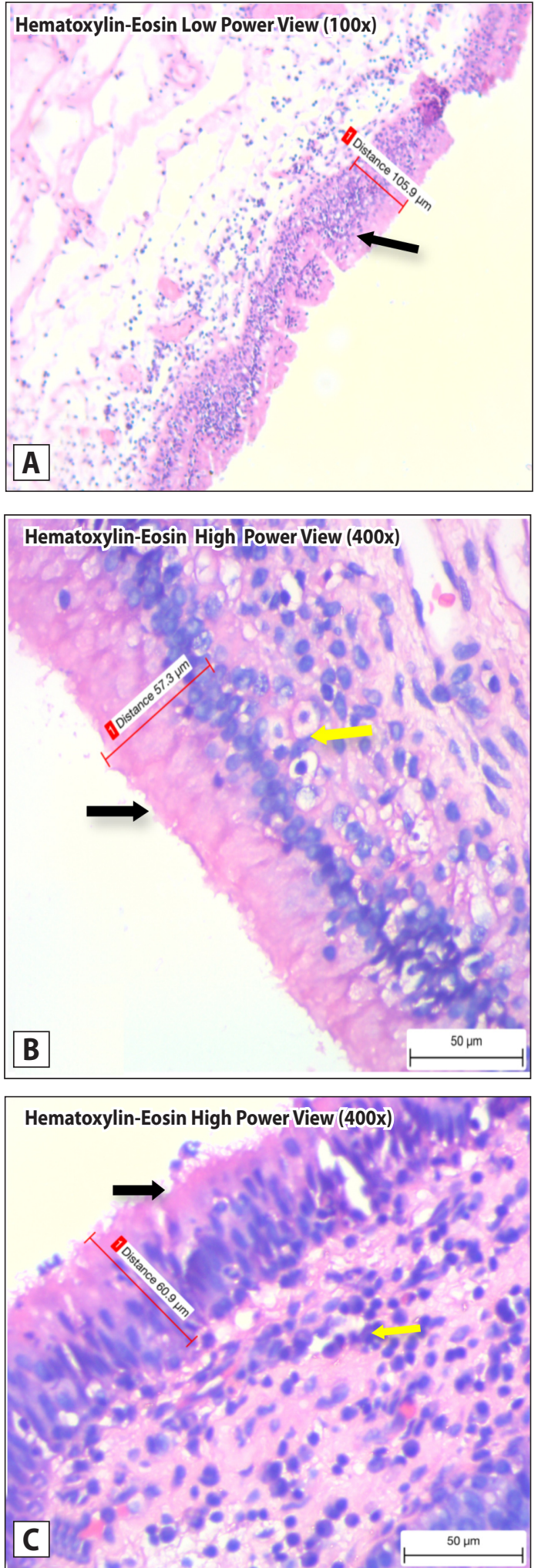

Figure 1. Photomicrographs, histopathological (H\&E) examination of nasal polyps from control group showing columnar epithelium (with measurement) overlaid by biofilm layer (black arrow). A. Low power view (100x). B \& C. High power view (400x) showing biofilm and submucosal lymphoplastic infiltrates (yellow arrow)
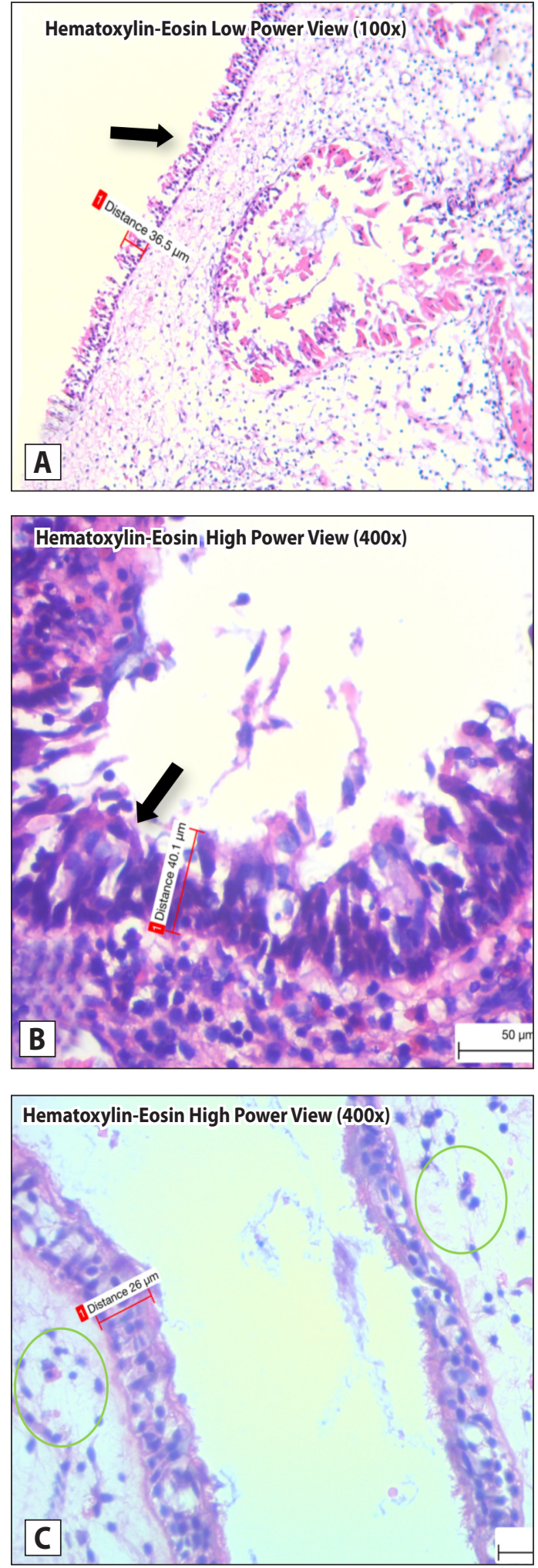

Figure 2. Photomicrographs, histopathological (H\&E) examinations of nasal polyps from treatment group showing columnar epithelium (with measurement) without/disrupted biofilm layer (black arrow). A. Low power view (100x). B. High power view (400x) C. High power 


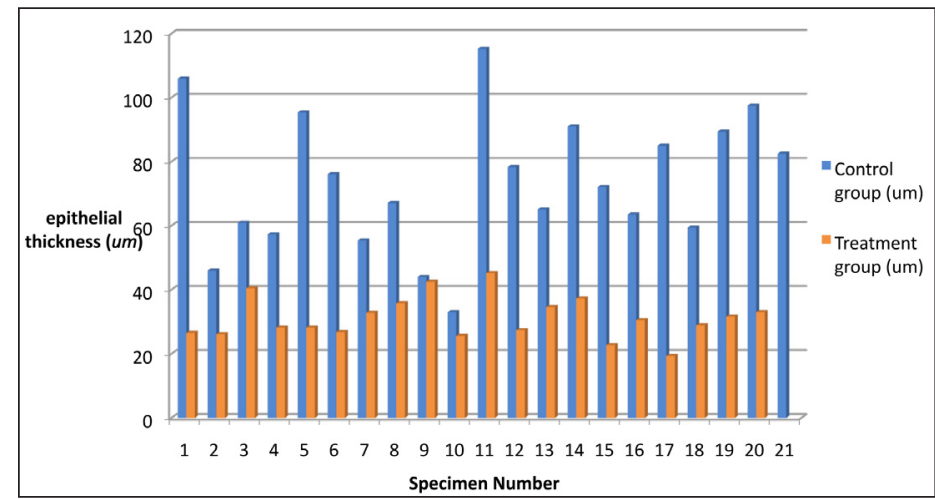

Figure 3. Comparison of epithelial thickness of nasal polyps (surface epithelium + biofilm matrix)

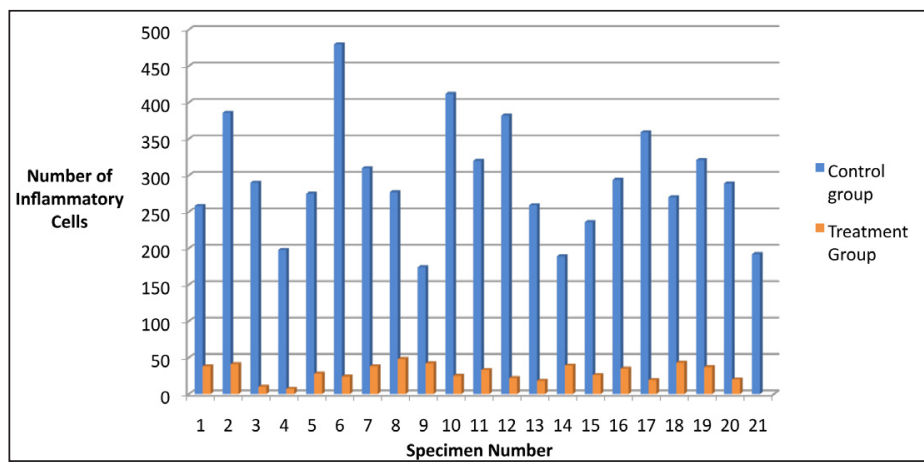

Figure 4. Comparison of average number of inflammatory cells per high power field in 10 random sites

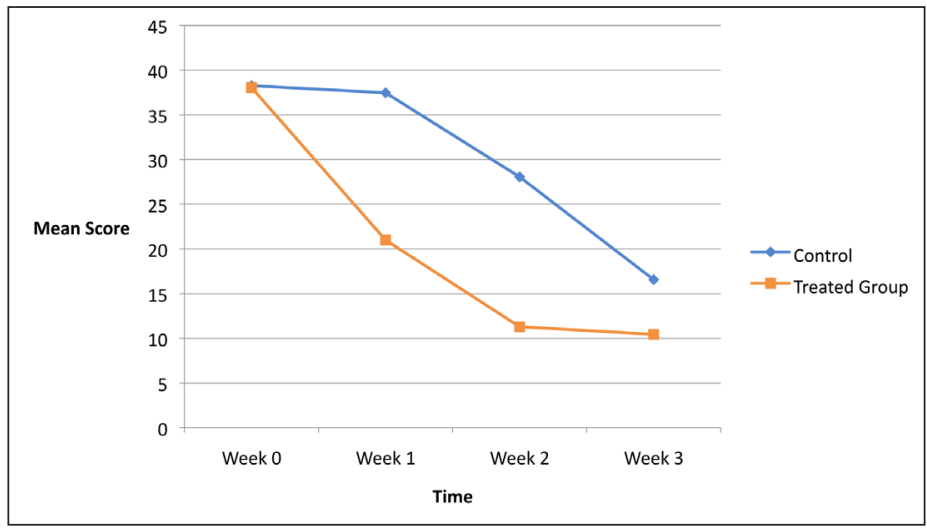

Figure 5. Comparison of SNOT-22 mean scores between control and ultrasound treated groups

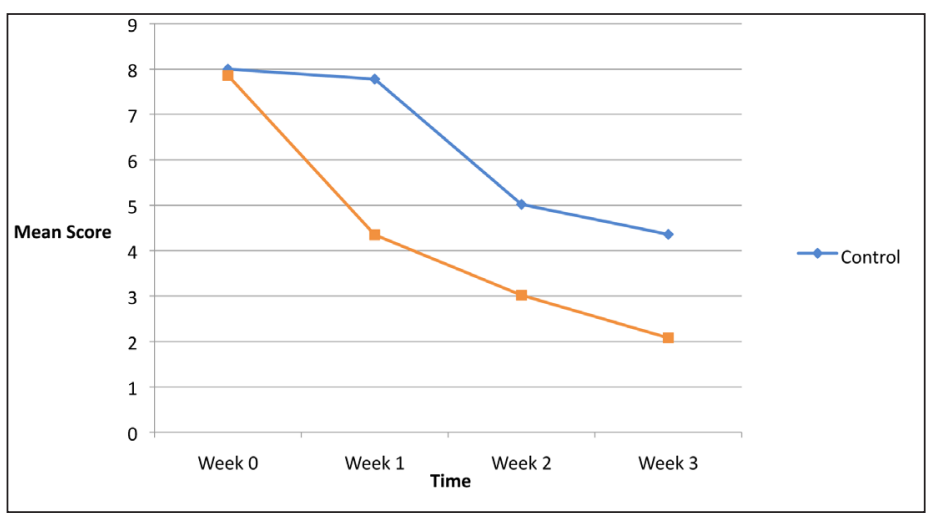

Figure 6. Comparison of modified Lund MacKay endoscopic appearance scores between control and ultrasound treated groups $(p=2.052 \mathrm{E}-32 ; 9.26 \mathrm{E}-30 ; \mathrm{Cl} 95 \%)$ between the two groups. This suggests that improvement from ESS in the ultrasound treated group was much better and faster compared to the control group. (Figure 5) This result was further strengthened by two-way analysis of variance that showed significant differences between the two groups with respect to treatment and time $(p=1.07 \mathrm{E}-80 ; 9.71 \mathrm{E}-$ 119). (Table 2)

Modified Lund-MacKay Endoscopic Assessment mean scores for the control group in weeks 0 to week 3 were 8 (SD 0.61), 7.78 (SD 0.54), 5.02 (SD 0.37), and 4.36 (SD 0.32), respectively. For the ultrasound treated group, mean results were 7.86 (SD 0.58), 4.35 (SD 0.46), 3.02 (SD 0.30), and 2.08 (SD 0.29), respectively. Analyzing results from the control group with respect to time, student T-test showed statistically significant changes only at week 2 ( $p=6.04 \mathrm{E}-22 ; \mathrm{Cl} 95 \%)$ and week 3 $(p=6.04 \mathrm{E}-22 ; \mathrm{Cl} 95 \%)$, while results from the ultrasound treated group showed statistically significant differences starting from week 1 to week 3 scores ( $p=1.14 \mathrm{E}-22,4.57 \mathrm{E}-13,2.73 \mathrm{E}-12$ ). (Table 3) Comparing both groups, student T-test showed a statistically significant difference in week 1 to week 3 with the greatest difference between week 1 and week 2 of treatment $(p=1.62 E-23)$. (Figure 6) Two-way ANOVA showed also significant difference between the two groups with respect to treatment and time $(p=3.89 \mathrm{E}-60 ; 1.85 \mathrm{E}-95)$. (Table 4)

\section{DISCUSSION}

Generally, therapeutic ultrasound is defined as frequency between 0.7-3.3 MHz with maximum energy absorption at a depth of 2 to 5 $\mathrm{cm}$ of soft tissue. ${ }^{8}$ In addition, attenuation of ultrasound through skin is approximately $2.7 \mathrm{~cm} .{ }^{9}$ This study used $1 \mathrm{MHz}$ which is within therapeutic range and $2 \mathrm{~cm}$ distance of the transducer from the polyp to mimic the approximate distance from the skin to the nasal cavity and sinuses. All these are in conformity with the therapeutic parameters proposed by Cameron. ${ }^{8}$

As ultrasound needs a medium through which sound waves will freely pass to reach the tissue, we used $\mathrm{NaCl}$ as a coupling medium in phase 1 and ultrasound gel in phase 2. They are ideal coupling media that fill all available spaces and are viscous enough to allow energy transmission with minimal absorption, attenuation, or disturbance. ${ }^{9}$

Phase 1 of the study centered on biofilm detection. The gold standard for biofilm detection is still electron microscopy but several studies demonstrated good correlation of Hematoxylin and Eosin staining in detection of biofilm in CRS. ${ }^{5}$ Watelet et al. stated that basement membrane thickening, a sign of chronic inflammation and airway remodeling and seromucinous gland abundance were displayed mostly in nasal polyps. ${ }^{12}$ Though the enumerated criteria for histopathological correlation of biofilm detection were not all met, results from this study may suggest a possible role of low-frequency ultrasound on the epithelial thickness and number of inflammatory cells between control and treatment groups. The difference in epithelial thickness may be attributed to conversion of the absorbed ultrasound 


\section{ORIGINAL ARTICLES}

Philippine Journal Of Otolaryngology-Head And Neck Surgery

VOL. 32 No. 1 JANUARY - JUNE 2017

PIOHNS

Table 1. Comparison of SNOT-22 total mean scores between control and ultrasound treated groups

\begin{tabular}{|l|c|c|c|c|c|c|c|c|c|} 
& \multicolumn{2}{|c|}{ Week 0 } & \multicolumn{2}{c|}{ Week 1 } & \multicolumn{3}{c|}{ Week 2 } & Week3 \\
& control & ultrasound & control & ultrasound & control & ultrasound & control & ultrasound \\
\hline Total & 804 & 761 & 787 & 420 & 590 & 226 & 348 & 209 \\
\hline Mean & 38.26 & 38.05 & 37.47 & 21 & 28.06 & 11.3 & 16.57 & 10.45 \\
\hline Standard deviation & 1.62 & 1.96 & 1.36 & 1.41 & 1.73 & 1.59 & 1.78 & 1.19 \\
\hline p-value & & & 0.09 & $7.24 \mathrm{E}-29$ & $4.76 \mathrm{E}-22$ & $4.80 \mathrm{E}-22$ & $2.62 \mathrm{E}-23$ & 0.06 \\
\hline
\end{tabular}

Table 2. Analysis of Variance (2-way ANOVA) of weekly (4 weeks) SNOT-22 Scores between control and ultrasound treated groups

\begin{tabular}{|l|r|r|c|c|c|c|}
$\begin{array}{l}\text { Source of } \\
\text { Variation }\end{array}$ & SS & df & MS & F & P-value & F crit \\
\hline Sample & 3890.756 & 1 & 3890.756 & 1502.719 & $1.07 \mathrm{E}-80$ & 3.903366 \\
\hline Columns & 14087.87 & 3 & 4695.956 & 1813.709 & $9.7 \mathrm{E}-119$ & 2.664107 \\
\hline Interaction & 1998.069 & 3 & 666.0229 & 257.2366 & $2.49 \mathrm{E}-{ }^{59}$ & 2.664107 \\
\hline Within & 393.55 & 152 & 2.589145 & & & \\
\hline & & & & & & \\
\hline Total & 20370.24 & 159 & & & & \\
\hline
\end{tabular}

Table 4. Analysis of Variance (2-way ANOVA) of Weekly (4 weeks) Lund MacKay endoscopic appearance scores between control and ultrasound treated groups

\begin{tabular}{|l|r|r|r|r|r|c|}
\hline $\begin{array}{l}\text { Source of } \\
\text { Variation }\end{array}$ & SS & df & MS & F & P-value & F crit \\
\hline Sample & 153.0766 & 1 & 153.0766 & 736.0265 & $3.89 \mathrm{E}-60$ & 3.903366 \\
\hline Columns & 542.8797 & 3 & 180.9599 & 870.0958 & $1.85 \mathrm{E}-95$ & 2.664107 \\
\hline Interaction & 56.86719 & 3 & 18.95573 & 91.1434 & $8.51 \mathrm{E}^{-34}$ & 2.664107 \\
\hline Within & 31.6125 & 152 & 0.207977 & & & \\
\hline & & & & & & \\
\hline Total & 784.4359 & 159 & & & & \\
\hline
\end{tabular}

Table 3. Comparison of modified Lund MacKay endoscopic appearance scores between control and ultrasound treated groups

\begin{tabular}{|l|c|l|c|c|c|c|c|c|c|} 
& \multicolumn{2}{|c|}{ Week 0 } & \multicolumn{2}{c|}{ Week 1 } & \multicolumn{3}{c|}{ Week 2 } & Week3 \\
& control & ultrasound & control & ultrasound & control & ultrasound & control & ultrasound \\
\hline Total & 168 & 157.5 & 163.5 & 87 & 105.5 & 60.5 & 91.5 & 41.5 \\
\hline Mean & 8 & 7.875 & 7.78 & 4.35 & 5.02 & 3.025 & 4.36 & 2.075 \\
\hline Standard deviation & 0.61 & 0.58 & 0.54 & 0.46 & 0.37 & 0.30 & 0.32 & 0.29 \\
\hline p-value & & & 0.24 & $1.14 \mathrm{E}-22$ & $6.04 \mathrm{E}-22$ & $1.14 \mathrm{E}-22$ & $2.25 \mathrm{E}-07$ & $2.73 \mathrm{E}-12$ \\
\hline
\end{tabular}

energy to mechanical energy that led to destruction of the outermost layer of epithelium. This can be observed from the photomicrograph examination (Figure 1) of nasal polyps from control group showing columnar epithelium overlaid by an outer layer made of dense extracellular polysaccharide substance (EPS) material compared to the sample from the treatment group showing no EPS layer on top of the columnar epithelium. (Figure 2) The mechanical disturbance caused by alternating rarefraction and compression of ultrasound may have affected diffusion rates and membrane permeability of sodium and calcium ions resulting in changes in the cell membrane potential and altered protein synthesis leading to biofilm instability and subsequent damage. ${ }^{9}$ This effect of ultrasound may also account for the decreased inflammatory cell count in the stromal and submucosal layer in the ultrasound treated group (Figure 2) compared with the control group showing predominance of lymphoplastic infiltrates in the submucosal layer. (Figure 1) Others explained this phenomenon secondary to increasing hydrophobic molecular transport through cell membrane, bacterial cell wall damage and loss of calcium in biofilm matrix causing instability and subsequent damages. ${ }^{13}$ This is consistent with results in an experimental study by Karosi et al. ${ }^{14}$ in which the ultrasound treated group showed disruption of biofilm layer with significant decrease in inflammatory cell count in the epithelial and stromal layer. Ensing et al. ${ }^{15}$ demonstrated reduction of planktonic and biofilm viability of E.coli, P. aeruginosa, S. aereus and coagulase negative staphylococci after gentamycin treatment following prolonged ultrasound treatment.

Phase II results of the study showed faster recovery of patients in the 
treatment group after ESS as shown on SNOT-22 mean weekly reports, supported by objective assessment via modified Lund Mackay mean results. Though not established in this study, these results may also be related to the mechanical effect of pulsed ultrasound in reducing inflammation, loosening stagnant secretions and possibly facilitating sinus drainage. ${ }^{16}$ Naghdi et al. studied 30 CRS adults who underwent 10 sessions of continuous therapeutic ultrasound, with percentage improvements of $74 \%$ and $72 \%$ at the end of the treatment and one month after treatment. ${ }^{17}$ Ansari et al. used low intensity pulsed ultrasound in a case series of 57 CRS patients that showed significant changes of most major and minor symptoms of CRS after ultrasound therapy. ${ }^{18}$

With respect to wound healing, faster recovery of patients under the ultrasound group with marked decreased of granulation, edema, crusting compared to the control group as early as week 1 of treatment suggest a possible role of ultrasound as an inflammatory optimizer. A study by Harvey et al. demonstrated that low dose pulsed ultrasound increases protein synthesis and enhanced fibroplasia and collagen synthesis in human fibroblasts. ${ }^{19}$ Rocha et al. reported that $1 \mathrm{MHz}$ low intensity ultrasound at $1 \mathrm{~W} / \mathrm{cm} 2$ for 4 minutes over maxillary sinus and nasal septum not only reduced nasal block and congestion by $64 \%$ but also resulted in regression in major symptoms of CRS. ${ }^{20}$

One unexpected finding in this study was squamous metaplasia in two specimens in the control group. A study by Baird et al. ${ }^{21}$ of 20 patients with nasal polyps found that $50 \%$ showed squamous metaplasia. They attributed this finding to chronicity of the disease. This was corroborated by the study of Shulbha et al. ${ }^{22}$ which showed $16 \%$ of 91 patients with nasal polyposis had squamous metaplasia, suggesting it is more common than previously thought.

One weakness of this study is that Hematoxylin and Eosin findings of biofilm presence were not confirmed by standard methods. Also, a double blinding would have been more appropriate in minimizing evaluator bias. The clinical portion of this study was limited by its small sample size and short duration of follow up. Future clinical trials may investigate intranasal application of ultrasound as part of medical management of CRS with grade 1 polyps to further establish the effect of low intensity therapeutic ultrasound on nasal polyps cellular architecture and on inflammation and recovery after ESS. In time, it may prove to be a valuable adjuvant treatment to conventional medical therapy for Chronic Rhinosinusitis with Nasal Polyposis and be recommended to improve recovery after Endoscopic Sinus Surgery.

There was no adverse effect to the use of therapeutic low frequency ultrasound reported in this study. Comparing the recovery between the two groups, the ultrasound group showed early and marked improvement in CRS symptoms and improved endoscopic appearance after Endoscopic Sinus Surgery. The antiinflammatory and antimicrobial effects of low frequency ultrasound are well documented in vitro, but its in vivo effects need further investigation. Our results suggest the possible use of low frequency therapeutic ultrasound in disrupting epithelial barriers or extracellular polysaccharide substance (EPS of biofilm) in patients with CRS with Nasal Polyposis as well as in recovery after Endoscopic Sinus Surgery based on SNOT-22 and modified Lund MacKay endoscopic appearance scores.

\section{REFERENCES}

1. Antonio T, Hernandez J, Lim M, Mangahas L, Quiambao R, Tan GP, et al. Diagnosis and Management of Chronic Rhinosinusitis in Adults. In Jose EM, Caro RM, Acuin JM (editors). PSO-HNS Clinical Practice Guidelines on Otitis Media, Rhinitis, Sinusitis, Tonsilitis, Obstructive Adenoidal Hypertrophy. Quezon City; PSO-HNS, Inc. 1997. Pp. 11-20.

2. Bachert C, Pawankar R, Zhang L, Bunnag C, Fokkens W, Hamilos D, et al. ICON: chronic rhinosinusitis. World Allergy Organ J. 2014 Oct 27; 7(1):25. [cited 2014 Nov]. Available from: http://www.waojournal.org/content/7/1/25. DOI: 10.1186/1939-4551-7-25; PMID: 25379119 PMCID: PMC4213581

3. Chambless JD, Hunt SM, Stewart PS. AThree-dimensional Computer Model of Four Hypothetical Mechanisms Protecting Biofilms from Antimicrobials. Appl Environ Microbiol. 2006 Mar; 72 (3):2005-2013. DOI: 10.1128/AEM.72.3.2005-2013.2006; PMCID: PMC1393201.

4. Fellers TJ, Davidson MW. Introduction to Confocal Microscopy. Olympus Fluoview Resource Center. National High Magnetic Field Laboratory. [Internet] Florida; c2004-2009. [Retrieved $2007 \mathrm{Jul}$ 25]. Available from: http://fluoview.magnet.fsu.edu/theory/confocalintro.html.

5. Hong SD, Dhong HJ, Chung SK, Kim HY, Park J, Ha SY. Hematoxylin and eosin staining for detecting biofilms: practical and cost-effective methods for predicting worse outcomes after endoscopic sinus surgery. Clin Exp Otorhinolaryngol. 2014 Sep; 7(3): 193-197. DOI: 10.3342 ceo.2014.7.3.193; PMID: 25177435 PMCID: PMC4135155.

6. Naghdi S, Ansari N, Farhadi M. A clinical trial on the treatment of chronic rhinosinusitis with continuous ultrasound. J Phys Ther Sci. 2008; 20(4):233-238.

7. Al-Haddad M, Al-Jumaily A, Brooks J, Bartley J. Biophysical Effects on Chronic Rhinosinusitis Bacterial Biofilms. In: Mahboub BH (editor). Respiratory Disease and Infection - A New Insight 2013. Atlanta Georgia: Intech. p.79-97. DOI: 10.5772/53860.

8. Cameron MH. Physical Agents in Rehabilitation (from research to practice). 4th ed. St Louis: Saunders; 2013.

9. Watson T. Ultrasound in contemporary physiotherapy practice. Ultrasonics. 2008 Aug; 48(4): 321-329. DOI: 10.1016/j.ultras.2008.02.004; PMID: 18466945.

10. Hsieh YL. Reduction in induced pain by ultrasound may be caused by altered expression of spinal neuronal nitric oxide synthase producing neurons. Arch Phys Med Rehabil. 2005 Jul; 86(7):1311-1317. PMID: 16003656.

11. Young $D$, Morton R, Bartley J. Therapeutic ultrasound as treatment for chronic rhinosinusitis: preliminary observations. J Laryngol Otol. 2010 May; 124(5):495-9. DOI: 10.1017/ S0022215109992519; PMID: 20053307.

12. Watelet JB, Bachert C, Claeys C, Van Cauwenberge P. Matrix metalloproteinases MMP-7, MMP-9 and their tissue inhibitor TIMP-1: expression in chronic sinusitis vs nasal polyposis. Allergy. 2004 Jan; 59(1): 54-60. PMID: 14674934

13. Mortimer AJ, Dyson M. The effect of therapeutic ultrasound on calcium uptake in fibroblast Ultrasound Med Biol. 1988; 14(6):449-506. PMID: 3227573.

14. Karosi T, Sziklai I, Csomor P. Low frequency ultrasound for biofilm disruption in chronic rhinosinusitis with nasal polyposis: in vitro pilot study. Laryngoscope. 2013 Jan; 123(1): 17-23. DOI: 10.1002/lary.23633; PMID: 22893599.

15. Ensing GT, Neut D, van Horn JR, van der Mei HC, Busscher HJ. The combination of ultrasound with antibiotics released from bone cement decreases the viability of planktonic and biofilm bacteria: an in vitro study with clinical strains. J Antimicrob Chemother. 2006 Dec; 58(6): 128790. DOI: 10.1093/jac/dkI402; PMID: 17041238.

16. Ansari NN, Fathali M, Naghdi S, Hasson S, Jalaie S, Rastak MS. A randomized, doubleblind clinical trial comparing the effects of continuous and pulsed ultrasound in patients with chronic rhinosinusitis. Physiother Theory Pract. 2012 Feb; 28(2):85-94. DOI: 10.3109/09593985.2011.571751; PMID: 21823980.

17. Naghdi S, Ansari N, Farhadi M. A Clinical Trial on the Treatment of Chronic Rhinosinusitis with Continuous Ultrasound. J Phys Ther Sci. 2008; 20:233-238.

18. Ansari NN, Naghdi S, Farhadi M, Jalaie S. A preliminary study into the effect of low-intensity pulsed ultrasound on chronic maxillary and frontal sinusitis. Physiother Theory Pract. $2007 \mathrm{Jul}$ Aug; 23(4):211-8. DOI: 10.1080/09593980701209360; PMID: 17687734.

19. Harvey W, Dyson M, Pond JB, Grahame R. The stimulation of protein synthesis in human fibroblasts by therapeutic ultrasound. Rheumatol Rehabil. 1975 Nov; 14(4): 237. PMID: 1198016

20. Rocha WA, Rodrigues KM, Pereira RR, Nogueira BV, Goncalves WL. Acute effects of therapeutic 1-mhz ultrasound on nasal unblocking of subjects with chronic rhinosinusitis. Braz J Otolaryngol. 2011 Jan-Feb; 77(1): 7-12. PMID: 21340182.

21. Baird AR, Hilmi O, White PS, Robertson AJ. Epithelial atypia and squamous metaplasia in nasal polyps. J Laryngol Otol. 1998 Aug; 112(8): 755-7. PMID: 9850317.

22. Shulbha VS, Dayananda BS. Clinicopathological study of nasal polyps with special references to the mast cells in inflammatory polyps. Basic Appl Pathol. 2012 Sep; 5(3): 59-62. DOI: 10.1111/ j.1755-9294.2012. 01136. 